\title{
Identifiability for Linearized Sine-Gordon Equation
}

\author{
J. Ha ${ }^{1}$, S. Gutman ${ }^{2} *$ \\ ${ }^{1}$ School of Liberal Arts, Korea University of Technology and Education \\ Cheonan 330-708, South Korea \\ 2 Department of Mathematics, University of Oklahoma \\ Norman, Oklahoma 73019, USA
}

\begin{abstract}
The paper presents theoretical and numerical results on the identifiability, i.e. the unique identification for the one-dimensional sine-Gordon equation. The identifiability for nonlinear sine-Gordon equation remains an open question. In this paper we establish the identifiability for a linearized sine-Gordon problem. Our method consists of a careful analysis of the Laplace and Fourier transforms of the observation of the system, conducted at a single point. Numerical results based on the best fit to data method confirm that the identification is unique for a wide choice of initial approximations for the sought test parameters. Numerical results compare the identification for the nonlinear and the linearized problems.
\end{abstract}

Keywords and phrases: Identification, identifiability, sine-Gordon equation

Mathematics Subject Classification: 35R30, 93B30

\section{Introduction}

Let $q=(\alpha, \beta, \gamma) \in P \subset \mathbb{R}^{3}$ be a triple of parameters. The corresponding damped one-dimensional sine-Gordon problem is

$$
\begin{array}{ll}
y_{t t}+2 \alpha y_{t}-\beta y_{x x}+\gamma \sin y=f(t, x), & (t, x) \in Q=(0, T) \times(0, \pi), \\
y(t, 0)=y(t, \pi)=0, & t \in(0, T), \\
y(0, x)=g(x), y_{t}(0, x)=h(x), & x \in(0, \pi) .
\end{array}
$$

Equation (1.1) describes the dynamics of a Josephson junction driven by a current source taking into account the damping effect [1]. This model has attracted an additional interest since it is known to exhibit chaotic behavior for certain values of the governing parameters $\alpha, \beta$ and $\gamma$. For details we refer to $[10]$ and [19].

We study the case of $f(t, x) \equiv 0$ in $Q$. Then the corresponding linearized sine-Gordon problem is

$$
\begin{array}{ll}
y_{t t}+2 \alpha y_{t}-\beta y_{x x}+\gamma y=0, & (t, x) \in Q=(0, T) \times(0, \pi), \\
y(t, 0)=y(t, \pi)=0, & t \in(0, T), \\
y(0, x)=g(x), y_{t}(0, x)=h(x), & x \in(0, \pi) .
\end{array}
$$

The linearized equation is important in physics and electronics. In fact, equation (1.2) is a linear KleinGordon equation describing the effect of the shed dispersive radiation on the evolving soliton [17]. In

\footnotetext{
${ }^{*}$ Corresponding author. E-mail: sgutman@ou.edu
} 
signal analysis of transmission and propagation of electrical signals equation (1.2) is referred to as a telegraph equation with constant coefficients, [12]. Necessary conditions for the coefficients $\alpha$ and $\gamma$ that imply periodic or bounded solutions of (1.1)-(1.2) are discussed in [14].

The parameter identification problem for (1.1) was discussed in [7]. The authors have established the existence of an optimal parameter triple $q^{*}=\left(\alpha^{*}, \beta^{*}, \gamma^{*}\right) \in P$ satisfying

$$
J\left(q^{*}\right)=\inf _{P} J(q)=\inf _{P}\left\|y(q)-z_{d}\right\|_{L^{2}(Q)}^{2},
$$

where $y(q)$ is the solution of $(1.1), z_{d} \in L^{2}(Q)$ is the targeted behavior, and a closed and convex set $P \subset \mathbb{R}^{3}$ is an admissible set. Furthermore, it was shown in [7] that such an optimal set of parameters $q^{*}$ can be characterized by a bang-bang type of optimality condition. In [6] this problem was studied from a computational perspective.

In this paper our goal is to present theoretical and numerical results on the identifiability, i.e. the unique identification for the one-dimensional linearized sine-Gordon problem. In general, there are many optimal parameters $q^{*}$ in the admissible set $P$. The identifiability problem is to determine under what conditions on the admissible set $P$ and the observations of the system (1.2) one can conclude that there is a unique optimal parameter set $q^{*}$. For various identifiability results see $[4,5,8,13,15]$ and $[9]$. The identifiability for the nonlinear sine-Gordon problem (1.1) is still an open question.

Our results show that under some mild conditions on the initial data in (1.2) the parameters $\alpha, \beta, \gamma$ are identifiable from an observation of the solution $y(t, x ; q), t \geq 0$ at a single point $p \in(0, \pi)$. The problem setup is given in section 2. Our main identifiability result Theorem 4.3 is presented in section 4. Computational algorithms are discussed in section 5. In section 6 we give numerical examples of the identifiability for (1.1) and (1.2) using a one-point observation data.

\section{Problem setup}

Let Hilbert space $H=L_{2}(0, \pi)$ have the norm $|\cdot|$ and the inner product $(\cdot, \cdot)$. Let $V=H_{0}^{1}(0, \pi)$ have the norm $\|\cdot\|$ and the inner product $((\cdot, \cdot))$. Denote by $V^{\prime}$ the dual space of $V$. We consider the standard triple $V \subset H \subset V^{\prime}$, where the inner product $(\cdot, \cdot)$ extends to the duality mapping between $V$ and $V^{\prime}$.

The parameters $\alpha, \beta, \gamma$ are required to be in the admissible set

$$
P=\left\{(\alpha, \beta, \gamma): \alpha \geq 0, \beta \geq \beta_{\min }>0, \gamma \geq 0\right\} .
$$

Within this framework problem (1.2) is restated as an abstract differential equation

$$
y^{\prime \prime}+2 \alpha y^{\prime}+A y=0, \quad y(0)=g \in V, \quad y^{\prime}(0)=h \in H,
$$

where the operator $A$ is defined by the relation $(A y, v)=\beta\left(y_{x}, v_{x}\right)+\gamma(y, v)$ for $v \in V$. According to [3], [11], problem (2.2) has a unique weak solution $y \in C([0, T] ; V)$.

We need a more detailed information about the solution $y(t, x)$. First, we introduce some notation.

Definition 2.1. Let $q=(\alpha, \beta, \gamma) \in P$. Define the following subsets of $\mathbb{N}=\{1,2, \ldots\}$

$$
\Pi_{-}=\left\{k \in \mathbb{N}: k^{2} \beta+\gamma-\alpha^{2}<0\right\}
$$

$$
\Pi_{0}=\left\{k \in \mathbb{N}: k^{2} \beta+\gamma-\alpha^{2}=0\right\},
$$

$$
\Pi_{+}=\left\{k \in \mathbb{N}: k^{2} \beta+\gamma-\alpha^{2}>0\right\} .
$$

Note that $\Pi_{-}$is at most finite, $\Pi_{0}$ contains at most one element, and $\Pi_{+}$always contains infinitely many elements. Depending on the triple $(\alpha, \beta, \gamma) \in P$ it may happen that $\Pi_{-}$and $\Pi_{0}$ are empty. 
Theorem 2.2. Let $D=[0, \infty) \times[0, \pi]$, and $w_{k}(x)=\sqrt{\frac{2}{\pi}} \sin k x, k=1,2, \ldots$ Then

(i) The weak solution $y(t, x)$ of $(2.2)$ on $D$ is given by

$$
\begin{aligned}
y(t, x)=e^{-\alpha t} \sum_{k \in \Pi_{-}}\left(A_{k} e^{\alpha_{k} t}+B_{k} e^{-\alpha_{k} t}\right) w_{k}(x) & \\
& +e^{-\alpha t}\left(A_{m}+t B_{m}\right) w_{m}(x)+e^{-\alpha t} \sum_{k \in \Pi_{+}}\left(A_{k} \cos \alpha_{k} t+B_{k} \sin \alpha_{k} t\right) w_{k}(x),
\end{aligned}
$$

where $\alpha_{k}, A_{k}, B_{k}$ are given as follows:

If $k \in \Pi_{-}$, then $\alpha_{k}=\sqrt{\alpha^{2}-\beta k^{2}-\gamma}$,

$$
\begin{aligned}
& A_{k}=\frac{1}{2}\left(1+\frac{\alpha}{\alpha_{k}}\right)\left(g, w_{k}\right)+\frac{1}{2 \alpha_{k}}\left(h, w_{k}\right), \\
& B_{k}=\frac{1}{2}\left(1-\frac{\alpha}{\alpha_{k}}\right)\left(g, w_{k}\right)-\frac{1}{2 \alpha_{k}}\left(h, w_{k}\right) .
\end{aligned}
$$

If $\Pi_{0}=\{m\}, m \in \mathbb{N}$, then $A_{m}=\left(g, w_{m}\right)$, and $B_{m}=\alpha A_{m}+\left(h, w_{m}\right)$.

If $k \in \Pi_{+}$, then $\alpha_{k}=\sqrt{\beta k^{2}+\gamma-\alpha^{2}}$,

$$
A_{k}=\left(g, w_{k}\right), \quad B_{k}=\frac{1}{\alpha_{k}}\left[\alpha A_{k}+\left(h, w_{k}\right)\right] .
$$

(ii) The series

$$
v(t, x)=\sum_{k \in \Pi_{+}}\left(A_{k} \cos \alpha_{k} t+B_{k} \sin \alpha_{k} t\right) w_{k}(x)
$$

converges uniformly on $D$. Function $v(t, x)$ is a continuous and bounded function on $D$.

(iii) The series in (2.3) converges uniformly on $D$. The solution $y(t, x)$ is a continuous and bounded function on $D$.

Proof. Since functions $\left\{w_{k}\right\}_{k=1}^{\infty}$ form an orthonormal basis in $H$, we can define Galerkin approximations $y^{N}(t, x)$ to the solution $y(t, x)$ of $(2.2)$ to be functions of the form

$$
y^{N}(t, x)=\sum_{k=1}^{N} b_{k}(t) w_{k}(x)
$$

satisfying conditions

$$
\begin{gathered}
\left(\frac{d^{2}}{d t^{2}} y^{N}+2 \alpha \frac{d}{d t} y^{N}+A y^{N}, w_{k}\right)=0 \\
\left(y^{N}(0), w_{k}\right)=\left(g, w_{k}\right), \quad\left(\frac{d}{d t} y^{N}(0), w_{k}\right)=\left(h, w_{k}\right)
\end{gathered}
$$

for $k=1,2, \ldots, N$. Therefore

$$
b_{k}^{\prime \prime}+2 \alpha b_{k}^{\prime}+\beta k^{2} b_{k}+\gamma b_{k}=0,
$$

and we conclude that $y^{N}(t, x)$ is given by the partial sum of the series (2.3) with $k \leq N$. Furthermore, the coefficients $A_{k}$ and $B_{k}, k=1,2 \ldots, N$ are defined as in (i) of the Theorem.

Multiply both sides in (2.6) by $b_{k}^{\prime}$. Then this equation can be rewritten as

$$
\frac{1}{2} \frac{d}{d t}\left[\left|b_{k}^{\prime}\right|^{2}+\left(\beta k^{2}+\gamma\right)\left|b_{k}\right|^{2}\right]+2 \alpha\left|b_{k}^{\prime}\right|^{2}=0
$$


Therefore

$$
\left|b_{k}^{\prime}\right|^{2}+\left(\beta k^{2}+\gamma\right)\left|b_{k}\right|^{2}+4 \alpha \int_{0}^{t}\left|b_{k}^{\prime}(s)\right|^{2} d s=\left|\left(h, w_{k}\right)\right|^{2}+\left(\beta k^{2}+\gamma\right)\left|\left(g, w_{k}\right)\right|^{2} .
$$

Since

$$
\left\|y^{N}\right\|^{2}=\left|\sum_{k=1}^{N} b_{k}(t) k \sqrt{\frac{2}{\pi}} \cos (k x)\right|_{H}^{2}=\sum_{k=1}^{N}\left|b_{k}\right|^{2} k^{2},
$$

and

$$
\|g\|^{2}=\sum_{k=1}^{\infty} k^{2}\left|\left(g, w_{k}\right)\right|^{2}
$$

equation (2.8) implies

$$
\left|\frac{d y^{N}}{d t}\right|^{2}+\beta\left\|y^{N}\right\|^{2}+\gamma\left|y^{N}\right|^{2}+4 \alpha \int_{0}^{t}\left|\frac{d y^{N}(s)}{d s}\right|^{2} d s \leq|h|^{2}+\beta\|g\|^{2}+\gamma|g|^{2} .
$$

Now the convergence of the Galerkin approximations $y^{N}$ to the solution $y$ defined in (2.3) can be established using energy estimate (2.11) as in [11] or [19]. Passing to the limit in (2.11), and accounting for $(2.8)$ gives

$$
\left|\frac{d y}{d t}\right|^{2}+\beta\|y\|^{2}+\gamma|y|^{2}+4 \alpha \int_{0}^{t}\left|\frac{d y(s)}{d s}\right|^{2} d s=|h|^{2}+\beta\|g\|^{2}+\gamma|g|^{2} .
$$

Equation (2.12) implies the uniqueness of the solution $y$.

To prove assertion (ii) of the Theorem we need a more detailed information about the coefficients $A_{k}, B_{k}, k \in \Pi_{+}$.

Since $A_{k}=\left(g, w_{k}\right)$ for $k \in \Pi_{+}$we have

$$
\sum_{k \in \Pi_{+}}\left|A_{k}\right|^{2} \leq|g|^{2}, \quad \text { and } \quad \sum_{k \in \Pi_{+}} k^{2}\left|A_{k}\right|^{2} \leq\|g\|^{2} .
$$

The coefficients $B_{k}$ are defined by $B_{k}=\frac{1}{\alpha_{k}}\left[\alpha A_{k}+\left(h, w_{k}\right)\right], k \in \Pi_{+}$Therefore, according to (2.4)

$$
\begin{aligned}
|v(t, x)| \leq \sqrt{\frac{2}{\pi}} \sum_{k \in \Pi_{+}}\left(\left|A_{k}\right|+\left|B_{k}\right|\right) & =\sqrt{\frac{2}{\pi}} \sum_{k \in \Pi_{+}}\left(k\left|A_{k}\right| \frac{1}{k}+\alpha_{k}\left|B_{k}\right| \frac{1}{\alpha_{k}}\right) \\
& \leq \frac{1}{\sqrt{2 \pi}} \sum_{k \in \Pi_{+}}\left(k^{2}\left|A_{k}\right|^{2}+\frac{1}{k^{2}}\right)+\frac{1}{\sqrt{2 \pi}} \sum_{k \in \Pi_{+}}\left(\alpha_{k}^{2}\left|B_{k}\right|^{2}+\frac{1}{\alpha_{k}^{2}}\right) .
\end{aligned}
$$

The first series in (2.14) is convergent because of (2.13). For the second series we have

$$
\sum_{k \in \Pi_{+}} \alpha_{k}^{2}\left|B_{k}\right|^{2}=\sum_{k \in \Pi_{+}}\left[\alpha A_{k}+\left(h, w_{k}\right)\right]^{2} \leq 2 \alpha^{2} \sum_{k \in \Pi_{+}}\left|A_{k}\right|^{2}+2 \sum_{k \in \Pi_{+}}\left|\left(h, w_{k}\right)\right|^{2} \leq 2 \alpha^{2}|g|^{2}+2|h|^{2},
$$

and

$$
\sum_{k \in \Pi_{+}} \frac{1}{\alpha_{k}^{2}}=\sum_{k \in \Pi_{+}} \frac{1}{\beta k^{2}+\gamma-\alpha^{2}}<\infty .
$$

Estimates (2.14)-(2.16) show that the series defining $v(t, x)$ in (2.4) converges uniformly on $D$ according to Weierstrass $M$-test. Thus $v(t, x)$ is a bounded continuous function on $D$ as claimed in the Theorem.

To establish (iii) of the Theorem we argue as follows. If $\alpha=0$, then $\Pi_{-}=\Pi_{0}=\emptyset$. So $y(t, x)=v(t, x)$ in this case. If $\alpha>0$, let $N=\max \left\{k \in \Pi_{-} \cup \Pi_{0}\right\}$. Then, by $(2.3)$, the approximation $y^{N}(t, \cdot):[0, \infty) \rightarrow$ $V$ is a continuous and bounded function on $[0, \infty)$. The $\Pi_{+}$part of the solution is $e^{-\alpha t} v(t, x)$, so the result follows from part (ii) of the Theorem. 


\section{Laplace and Fourier transforms of the observation $z_{p}(t)$}

Let $p \in(0, \pi)$. Then the observation of the solution $y(t, x), t \geq 0$ of the system (1.2) at the point $p$ is given by

$$
\begin{aligned}
z_{p}(t ; q)=y(t, p ; q)=e^{-\alpha t} & \sum_{k \in \Pi_{-}}\left(A_{k} e^{\alpha_{k} t}+B_{k} e^{-\alpha_{k} t}\right) w_{k}(p) \\
& +e^{-\alpha t}\left(A_{m}+t B_{m}\right) w_{m}(p)+e^{-\alpha t} \sum_{k \in \Pi_{+}}\left(A_{k} \cos \alpha_{k} t+B_{k} \sin \alpha_{k} t\right) w_{k}(p),
\end{aligned}
$$

where the coefficients $A_{k}$ and $B_{k}$ are defined in (i) of Theorem 2.2. By Theorem 2.2(iii) the observation $z_{p}(t ; q)$ is a bounded continuous function for $t \geq 0$.

The main tools in our arguments are the Laplace and the Fourier transforms of the observation $z_{p}(t)=$ $z_{p}(t ; q)$. While these transforms are related through a complex argument, it is more convenient for us to separate them.

The Laplace transform $\mathcal{L} f$ of a piecewise continuous function $f(t)$ satisfying $|f(t)| \leq M e^{a t}, t \geq 0$ for some $M, a \in \mathbb{R}$ is defined for any real $s>a$ by

$$
\mathcal{L} f(s)=\int_{0}^{\infty} e^{-s t} f(t) d t .
$$

The Fourier transform $\mathcal{F} f$ of a function $f \in L^{1}(\mathbb{R})$ is defined by

$$
\mathcal{F} f(w)=\frac{1}{\sqrt{2 \pi}} \int_{\mathbb{R}} f(t) e^{-i w t} d t, \quad w \in \mathbb{R} .
$$

It can be extended to tempered distributions $T \in \mathcal{S}^{\prime}(\mathbb{R})$ as described in [18], for which we refer for relevant definitions and results. In particular, we need the Fourier transform of the Heaviside function $H(t)$ defined by $H(t)=1$ for $t \geq 0$, and $H(t)=0$ for $t<0$. The Sokhatsky-Weierstrass theorem states that

$$
\mathcal{F}\{H(t)\}(w)=\frac{1}{\sqrt{2 \pi} i} p v\left(\frac{1}{w}\right)+\sqrt{\frac{\pi}{2}} \delta,
$$

where the distribution for $1 / w$ is defined by the principal value integral

$$
\left\langle p v\left(\frac{1}{w}\right), \phi(w)\right\rangle=\lim _{\epsilon \rightarrow 0+} \int_{|w|>\epsilon} \frac{\phi(w)}{w} d w, \quad \phi \in \mathcal{S}(\mathbb{R}),
$$

see [18], section 3.4 .

Lemma 3.1. Let $p \in(0, \pi), v(t, x)$ be defined by $(2.4)$, and $z_{p}(t ; q)=y(t, p ; q)$ be defined in (3.1). Then

(i) The Laplace transform of $v$ is given by

$$
V(s)=\mathcal{L}\{v(t, p)\}(s)=\sum_{k \in \Pi_{+}}\left(\frac{A_{k} s}{s^{2}+\alpha_{k}^{2}}+\frac{B_{k}}{s^{2}+\alpha_{k}^{2}}\right) w_{k}(p) .
$$

Function $V(s)$ is continuous and bounded for $s>0$.

(ii) The Laplace transform of $z_{p}$ is given by

$$
\begin{aligned}
Z(s)=\mathcal{L}\left\{z_{p}(t ; q)\right\}(s)=\sum_{k \in \Pi_{-}}\left(\frac{A_{k}}{s+\alpha-\alpha_{k}}+\frac{B_{k}}{s+\alpha+\alpha_{k}}\right) w_{k}(p) & \\
& +\left(\frac{A_{m}}{s+\alpha}+\frac{B_{m}}{(s+\alpha)^{2}}\right) w_{m}(p)+V(s+\alpha),
\end{aligned}
$$

where $m \in \Pi_{0}$. If $\Pi_{-} \neq \emptyset$, then function $Z(s)$ is defined for $s>-\alpha+\alpha_{1}$. Otherwise $Z(s)$ is defined for $s>-\alpha$. 
(iii) The Fourier transform of $v$ is given by

$$
\begin{aligned}
\mathcal{F} v(w)=-\frac{1}{2 \sqrt{2 \pi}} \sum_{k \in \Pi_{+}}^{\infty}\left\{A _ { k } i \left[p v\left(\frac{1}{w-\alpha_{k}}\right)+p v\right.\right. & \left(\frac{1}{w+\alpha_{k}}\right) \\
+\sqrt{\frac{\pi}{2}} \delta\left(w-\alpha_{k}\right)+\sqrt{\left.\frac{\pi}{2} \delta\left(w+\alpha_{k}\right)\right]} & +B_{k}\left[p v\left(\frac{1}{w-\alpha_{k}}\right)-p v\left(\frac{1}{w+\alpha_{k}}\right)\right. \\
& \left.\left.+\sqrt{\frac{\pi}{2}} \delta\left(w-\alpha_{k}\right)-\sqrt{\frac{\pi}{2}} \delta\left(w+\alpha_{k}\right)\right] w_{k}(p)\right\},
\end{aligned}
$$

where the principal value integrals are taken around the singularities.

(iv) For $\eta>0$ define

$$
u_{\eta}(t)= \begin{cases}e^{-\eta t} v(t, p), & t \geq 0 \\ 0, & t<0\end{cases}
$$

Then

$$
\mathcal{F} u_{\eta}(w)=\frac{1}{\sqrt{2 \pi}} \sum_{k \in \Pi_{+}}^{\infty} \frac{A_{k}(\eta+i w)+B_{k} \alpha_{k}}{\alpha_{k}^{2}+(\eta+i w)^{2}} w_{k}(p) .
$$

Function $\mathcal{F} u_{\eta}(w)$ is bounded for $w \in \mathbb{R}$.

(v) We have

$$
\lim _{\eta \rightarrow 0+} \mathcal{F} u_{\eta}=\mathcal{F} v
$$

as distributions.

Proof. The partial sums for $V(s)$ are obtained by taking the Laplace transform of the partial sums of $v(t, p)$ in (2.4). By Theorem 2.2 the series in (2.4) converges uniformly for $t \geq 0$, therefore expression (3.3) follows.

Let $\xi>0$. The maximum of the function $s \rightarrow s /\left(s^{2}+\xi^{2}\right)$ on $s \in[0, \infty)$ is attained at $s=\xi$, and the maximum value is $1 / 2 \xi$. Using estimates $(2.13)-(2.16)$ we get

$$
|V(s)| \leq \sum_{k \in \Pi_{+}}\left(\frac{\left|A_{k}\right| s}{s^{2}+\alpha_{k}^{2}}+\frac{\left|B_{k}\right|}{s^{2}+\alpha_{k}^{2}}\right)\left|w_{k}(p)\right| \leq \sqrt{\frac{2}{\pi}} \sum_{k \in \Pi_{+}}\left|A_{k}\right| \frac{1}{2 \alpha_{k}}+\sqrt{\frac{2}{\pi}} \sum_{k \in \Pi_{+}}\left|B_{k}\right| \frac{1}{\alpha_{k}^{2}}<\infty .
$$

Weierstrass $M$-test implies that $V(s)$ is bounded and continuous on $(0, \infty)$. Expression (3.4) follows from formula (3.1) for $z_{p}(t ; q)$, and (3.3).

By a direct computation

$$
\mathcal{F}\left\{e^{-\eta t} H(t)\right\}(w)=\frac{1}{\sqrt{2 \pi}} \frac{1}{\eta+i w} .
$$

Using $\mathcal{F}\left\{e^{i a t} f(t)\right\}=\mathcal{F} f(w-a)$,

$$
\mathcal{F}\left\{e^{-\eta t} H(t) \cos \alpha_{k} t\right\}(w)=\frac{1}{\sqrt{2 \pi}} \frac{1}{2}\left(\frac{1}{\eta+i\left(w-\alpha_{k}\right)}+\frac{1}{\eta+i\left(w+\alpha_{k}\right)}\right),
$$

and

$$
\mathcal{F}\left\{e^{-\eta t} H(t) \sin \alpha_{k} t\right\}(w)=\frac{1}{\sqrt{2 \pi}} \frac{1}{2 i}\left(\frac{1}{\eta+i\left(w-\alpha_{k}\right)}-\frac{1}{\eta+i\left(w+\alpha_{k}\right)}\right) .
$$

By Theorem 2.2 the series defining $v(t, x)$ in (2.4) converges uniformly on $D$. Therefore the series

$$
u_{\eta}(t)=\sum_{k \in \Pi_{+}}^{\infty}\left(A_{k} e^{-\eta t} H(t) \cos \alpha_{k} t+B_{k} e^{-\eta t} H(t) \sin \alpha_{k} t\right)
$$


defining $u_{\eta}(t)$ for $t \in \mathbb{R}$ converges uniformly on $\mathbb{R}$. Since the Fourier transform is a continuous operator in the space of tempered distributions $\mathcal{S}^{\prime}(\mathbb{R})$, we can apply it to this series termwise, and obtain (3.7).

By Theorem 2.2 function $v(t, p)$ is bounded for $0 \leq t<\infty$. Therefore $u_{\eta} \in L^{1}(\mathbb{R})$. This implies that its Fourier transform $\mathcal{F} u_{\eta}(w)$ is a continuous and bounded function for $w \in \mathbb{R}$.

Since $\lim _{\eta \rightarrow 0+} u_{\eta}(t)=v(t, p)$ uniformly on bounded intervals in $\mathbb{R}$, and the Fourier transform is continuous in $\mathcal{S}^{\prime}(\mathbb{R})$, we get (3.8). Accordingly, representation (3.5) can be obtained by passing to the limit in (3.7) as $\eta \rightarrow 0+$. However, this limit cannot be obtained by simply letting $\eta=0$ in (3.7). Indeed, letting $\eta=0$ in (3.10) gets an incorrect formula $\mathcal{F}\{H(t)\}(w)=1 /(\sqrt{2 \pi} i w)$, because the function $w \rightarrow 1 / w$ is not locally integrable in $\mathbb{R}$. The correct result for $\mathcal{F}\{H(t)\}(w)$ known as the Sokhatsky-Weierstrass theorem is stated in (3.2).

Therefore

$$
\begin{array}{r}
\mathcal{F}\left\{H(t) \cos \alpha_{k} t\right\}(w)=\frac{1}{\sqrt{2 \pi}} \frac{1}{2 i}\left[p v\left(\frac{1}{w-\alpha_{k}}\right)+p v\left(\frac{1}{w+\alpha_{k}}\right)\right. \\
\left.+\sqrt{\frac{\pi}{2}} \delta\left(w-\alpha_{k}\right)+\sqrt{\frac{\pi}{2}} \delta\left(w+\alpha_{k}\right)\right], \\
\mathcal{F}\left\{H(t) \sin \alpha_{k} t\right\}(w)=-\frac{1}{\sqrt{2 \pi}} \frac{1}{2}\left[p v\left(\frac{1}{w-\alpha_{k}}\right)-p v\left(\frac{1}{w+\alpha_{k}}\right)\right. \\
\left.+\sqrt{\frac{\pi}{2}} \delta\left(w-\alpha_{k}\right)-\sqrt{\frac{\pi}{2}} \delta\left(w+\alpha_{k}\right)\right],
\end{array}
$$

where the principal value integrals are taken around the singularities. Expression (3.5) follows.

Definition 3.2. Function $f: \mathbb{R} \rightarrow \mathbb{R}$ is called almost-periodic, if for any $\epsilon>0$ there exists $T=T(\epsilon)>0$ such that any interval $(t, t+T)$ contains at least one number $\tau$ such that $|f(y+\tau)-f(y)|<\epsilon$ for any $y \in \mathbb{R}$.

The main result in the theory of almost-periodic functions is that such functions are exactly the uniform limits of trigonometric polynomials $\sum_{k=1}^{m} c_{k} e^{i \alpha_{k} x}$, for complex $c_{k}$ and real $\alpha_{k}$, see $[2,20]$.

Let $v(t, x)$ be defined by (2.4). Then we have

Lemma 3.3. Extend function $v(t, p)$ to any $t \in \mathbb{R}$ by letting $t \in \mathbb{R}$ in $(2.4)$. Then $v(t, p)$ is an almostperiodic function on $\mathbb{R}$.

Proof. By Theorem 2.2(ii) function $v(t, x)$ is a uniform limit of trigonometric polynomials.

Lemma 3.4. Suppose that $\mu>0$, and $v(t, p) \not \equiv 0$ for $t \geq 0$. Then function $e^{\mu t} v(t, p)$ is unbounded on $[0, \infty)$.

Proof. Extend $v(t, p)$ to all $t \in \mathbb{R}$ by formula (2.4). Since $v(t, p)$ is continuous and assumed to be nonzero, there exists $y>0$ such that $v(y, p) \neq 0$. Let $\epsilon=|v(y, p)| / 2$. By Lemma 3.3 function $v(t, p)$ is almost periodic on $\mathbb{R}$. Let $T=T(\epsilon)>0$ be the corresponding number $T$ from the definition 3.2. Then there exists a sequence of numbers $\tau_{n} \in \mathbb{R}, n \in \mathbb{N}$ such that $\tau_{n} \in((n-1) T, n T)$, and $\left|v\left(y+\tau_{n}, p\right)-v(y, p)\right|<\epsilon$. We conclude that $\tau_{n} \rightarrow \infty$ as $n \rightarrow \infty$, and $\left|v\left(y+\tau_{n}, p\right)\right| \geq \epsilon / 2>0$. Therefore $e^{\mu\left(y+\tau_{n}\right)}\left|v\left(y+\tau_{n}, p\right)\right| \rightarrow \infty$, as $n \rightarrow \infty$, which means that function $e^{\mu t} v(t, p)$ is unbounded on $[0, \infty)$.

\section{Identifiability for linearized sine-Gordon problem}

The parameter identifiability for the system (1.2) in $P$ means that the assumption $q_{1}, q_{2} \in P$ and $z_{p}\left(t ; q_{1}\right)=z_{p}\left(t ; q_{2}\right)$ for all $t \geq 0$ implies $q_{1}=q_{2}$. We establish it by showing that the observation $z_{p}(t ; q), t \geq 0$ uniquely defines its generating triple of the parameters $q=(\alpha, \beta, \gamma) \in P$. 
In some cases, such as trivial initial data $g=h=0$, the observation $z_{p}(t) \equiv 0$ for any $q \in P$, and there is no hope to recover $q$ uniquely. Thus, we have to make some assumptions on the initial data. We choose either

$$
p \neq \frac{\pi}{2}, \quad\left(g, w_{1}\right)>0, \quad\left(h, w_{1}\right)>0, \quad\left(g, w_{2}\right) \neq 0, \quad\left(h, w_{2}\right) \neq 0,
$$

or

$$
p \neq \frac{\pi}{2}, \quad\left(g, w_{1}\right) \neq 0, \quad\left(g, w_{2}\right) \neq 0, \quad h=0 .
$$

We will always assume (without loss of generality) that $\left(g, w_{1}\right)>0$. The assumption $p \neq \pi / 2$, is used to assure that $w_{2}(p) \neq 0$. Since $w_{1}(x)>0$ on $(0, \pi)$, conditions $\left(g, w_{1}\right)>0,\left(h, w_{1}\right)>0$ can be achieved by taking $g(x)>0$ and $h(x)>0$ for $x \in(0, \pi)$. Conditions $\left(g, w_{2}\right) \neq 0$ and $\left(h, w_{2}\right) \neq 0$ are satisfied if functions $g$ and $h$ are not symmetric with respect to $x=\pi / 2$.

Recall that given $q \in P$, the corresponding subsets $\Pi_{-}, \Pi_{0}$, and $\Pi_{+}$were defined in Definition 2.1.

Lemma 4.1. Suppose that conditions (4.1) or (4.2) are satisfied. Let $q \in P, z_{p}(t ; q)=y(t, p ; q)$ for $t \geq 0$, and $Z(s)=\mathcal{L}\left\{z_{p}(t ; q)\right\}(s)$.

(i) If $\Pi_{-} \neq \emptyset$, then $1 \in \Pi_{-}$,

$$
\alpha>0, \quad \alpha_{1}=\sqrt{\alpha^{2}-\beta-\gamma}, \quad 0<\alpha_{1}<\alpha, \quad A_{1}>0, \quad B_{1}<0 .
$$

The Laplace transform $Z(s)$ is unbounded on interval $\left(-\alpha+\alpha_{1}, \infty\right)$.

(ii) If $\Pi_{-}=\emptyset$, but $\Pi_{0} \neq \emptyset$, then $\Pi_{0}=\{1\}, \Pi_{+}=\{2,3,4, \ldots\}$,

$$
\begin{gathered}
\alpha>0, \quad A_{1}>0, \quad B_{1}>0, \\
z_{p}(t ; q)=e^{-\alpha t}\left(A_{1}+t B_{1}\right) w_{1}(p)+e^{-\alpha t} v(t, p) .
\end{gathered}
$$

The Laplace transform $Z(s)$ is unbounded on interval $(-\alpha, \infty)$.

(iii) If $\Pi_{-}=\Pi_{0}=\emptyset$, then $\Pi_{+}=\{1,2,3, \ldots\}$,

$$
\begin{gathered}
\alpha \geq 0, \quad \alpha_{1}=\sqrt{\beta+\gamma-\alpha^{2}}>0, \quad A_{1}>0, \quad B_{1} \geq 0 . \\
z_{p}(t ; q)=e^{-\alpha t} v(t, p) .
\end{gathered}
$$

The Laplace transform $Z(s)$ is bounded on interval $(-\alpha, \infty)$.

Proof. Since $\beta>0$ and $\gamma \geq 0$, sets $\Pi_{-}$and $\Pi_{0}$ can be nonempty only if $\alpha>0$.

(i) If $\Pi_{-} \neq \emptyset$, then $\beta+\gamma-\alpha^{2}<0$. That is, $1 \in \Pi_{-}$. By Theorem 2.2(i), $\alpha_{1}=\sqrt{\alpha^{2}-\beta-\gamma}$. Thus $0<\alpha_{1}<\alpha$. By assumption $\left(g, w_{1}\right)>0$, and $\left(h, w_{1}\right) \geq 0$. Therefore

$$
A_{1}=\frac{1}{2}\left(1+\frac{\alpha}{\alpha_{1}}\right)\left(g, w_{1}\right)+\frac{1}{2 \alpha_{1}}\left(h, w_{1}\right)>0,
$$

and

$$
B_{1}=\frac{1}{2}\left(1-\frac{\alpha}{\alpha_{1}}\right)\left(g, w_{1}\right)-\frac{1}{2 \alpha_{1}}\left(h, w_{1}\right)<0 .
$$

By Lemma 3.1(ii), the Laplace transform $Z(s)$ is defined on $\left(-\alpha+\alpha_{1}, \infty\right)$. Its expression is given by (3.4). Lemma 3.1(i) shows that $V(s+\alpha)$ is bounded for $s \in\left(-\alpha+\alpha_{1}, \infty\right)$. All the other terms of $Z(s)$ are also bounded on $\left(-\alpha+\alpha_{1}, \infty\right)$, except for the very first term $A_{1} /\left(s+\alpha-\alpha_{1}\right)$. Since $A_{1} \neq 0$, this term is unbounded on $\left(-\alpha+\alpha_{1}, \infty\right)$. 
(ii) Suppose that $\Pi_{-}=\emptyset$, but $\Pi_{0} \neq \emptyset$. Then $\Pi_{0}=\{1\}$. Therefore $\Pi_{+}=\{2,3,4, \ldots\}$. Since $\Pi_{0} \neq 0$, we must have $\alpha>0$. From Theorem 2.2(i) we get the sought expression for $z_{p}(t ; q)$, and

$$
A_{1}=\left(g, w_{1}\right)>0, \quad B_{1}=\alpha A_{1}+\left(h, w_{1}\right)>0 .
$$

The Laplace transform $Z(s)$, given in Lemma 3.1)(ii), takes the form

$$
Z(s)=\left(\frac{A_{1}}{s+\alpha}+\frac{B_{1}}{(s+\alpha)^{2}}\right) w_{1}(p)+V(s+\alpha) .
$$

It is defined for $s>-\alpha$. By Lemma 3.1(i), function $V(s+\alpha)$ is bounded on $(-\alpha, \infty)$. The remaining term is unbounded on $(-\alpha, \infty)$, since $A_{1}, B_{1}>0$.

(iii) If $\Pi_{-}=\Pi_{0}=\emptyset$, then $k \in \Pi_{+}$for $k=1,2,3, \ldots$ In particular, $\beta+\gamma-\alpha^{2}>0$. By Theorem 2.2(i), $\alpha_{1}=\sqrt{\beta+\gamma-\alpha^{2}}>0$, and

$$
A_{1}=\left(g, w_{1}\right)>0, \quad B_{1}=\frac{1}{\alpha_{1}}\left[\alpha A_{1}+\left(h, w_{1}\right)\right] \geq 0 .
$$

The Laplace transform $Z(s)$ takes the form $Z(s)=V(s+\alpha)$. It is bounded on $(-\alpha, \infty)$ by Lemma 3.1(i).

Lemma 4.2. Suppose that conditions (4.1) or (4.2) are satisfied. Given $z_{p}(t)=y(t, p ; q) \not \equiv 0$, there exists $\xi \leq 0$ such that $\left\|e^{-\eta t} z_{p}(t)\right\|_{\infty}<\infty$ for $\eta>\xi$, and $\left\|e^{-\eta t} z_{p}(t)\right\|_{\infty}=\infty$ for $\eta<\xi$. If $\Pi_{-} \neq \emptyset$, then $\xi=\alpha_{1}-\alpha$. If $\Pi_{-}=\emptyset$, then $\xi=-\alpha$.

Proof. By Theorem 2.2(iii), $y(t, x ; q)$ is a bounded and continuous function on $D=[0, \infty) \times[0, \pi]$. Therefore $\xi \leq 0$. Also, by Theorem 2.2(ii), $\|v(t, p)\|_{\infty}<\infty$.

(i) If $\Pi_{-} \neq \emptyset$, then, by Lemma 4.1(i), $\alpha>0,0<\alpha_{1}<\alpha$, and $A_{1}>0$. Therefore, using expression (3.1) for the observation $z_{p}(t)$, we can write

$$
e^{-\eta t} z_{p}(t)=e^{\left(-\eta-\alpha+\alpha_{1}\right) t}\left(A_{1} w_{1}(p)+o(t)\right),
$$

where $o(t) \rightarrow 0$, as $t \rightarrow \infty$, with $A_{1} \neq 0$ and $w_{1}(p) \neq 0$.

If $\eta \geq \alpha_{1}-\alpha$, then $-\eta-\alpha+\alpha_{1} \leq 0$, and $\left\|e^{-\eta t} z_{p}(t)\right\|_{\infty}<\infty$. If $\eta<\alpha_{1}-\alpha$, then $-\eta-\alpha+\alpha_{1}>0$, and we have $\left\|e^{-\eta t} z_{p}(t)\right\|_{\infty}=\infty$. Thus we conclude that in this case $\xi=\alpha_{1}-\alpha$.

(ii) Suppose that $\Pi_{-}=\emptyset$, but $\Pi_{0} \neq \emptyset$. By Lemma 4.1(ii), $\Pi_{0}=\{1\}, B_{1}>0$, and $\alpha>0$. Then

$$
e^{-\eta t} z_{p}(t)=t e^{(-\eta-\alpha) t}\left(B_{1} w_{1}(p)+o(t)\right),
$$

where $o(t) \rightarrow 0$, as $t \rightarrow \infty$, with $B_{1} \neq 0$ and $w_{1}(p) \neq 0$.

If $\eta>-\alpha$, then $-\eta-\alpha<0$, and $\left\|e^{-\eta t} z_{p}(t)\right\|_{\infty}<\infty$. If $\eta<-\alpha$, then $\left\|e^{-\eta t} z_{p}(t)\right\|_{\infty}=\infty$. Thus, in this case $\xi=-\alpha$.

(iii) Suppose that $\Pi_{-}=\Pi_{0}=\emptyset$. By Lemma 4.1(iii),

$$
e^{-\eta t} z_{p}(t)=e^{(-\eta-\alpha) t} v(t, p) .
$$

Since $z_{p}(t) \not \equiv 0$, we have $v(t, p) \not \equiv 0$. If $\eta>-\alpha$, then $\left\|e^{\eta t} z_{p}(t)\right\|_{\infty}<\infty$, since $v(t, p)$ is bounded on $[0, \infty)$. If $\eta<-\alpha$, then $\left\|e^{\eta t} z_{p}(t)\right\|_{\infty}=\infty$ by Lemma 3.4. Thus, in this case $\xi=-\alpha$.

Our main result is the next Theorem. 
Theorem 4.3. Suppose that conditions (4.1) or (4.2) are satisfied. Given an observation $z_{p}(t ; q)=$ $y(t, p ; q) \not \equiv 0$ for $t \geq 0$, the parameters $q=(\alpha, \beta, \gamma)$ of the system (1.2) can be uniquely identified.

Proof. Let

$$
\xi=\inf \left\{\eta \in \mathbb{R}:\left\|e^{-\eta t} z_{p}(t)\right\|_{\infty}<\infty\right\} .
$$

By Lemma 4.2 such $\xi$ is finite, and $\left\|e^{-\eta t} z_{p}(t)\right\|_{\infty}<\infty$ for any $\eta>\xi$. This implies that the Laplace transform $Z(s)=\mathcal{L}\left\{z_{p}(t ; q)\right\}(s)$ exists for any $s>\xi$. Let

$$
b=\lim _{s \rightarrow \xi+} Z(s)(s-\xi)^{2} .
$$

Let us examine the boundedness of $Z(s)$ on $(\xi, \infty)$, and whether $b=0$, depending on the structure of the sets $\Pi_{-}, \Pi_{0}$, and $\Pi_{+}$.

If $\Pi_{-}=\Pi_{0}=\emptyset$, then $\xi=-\alpha$ by Lemma 4.2. By Lemma 4.1(iii), the Laplace transform $Z(s)$ is bounded on $(\xi, \infty)$.

If $\Pi_{-}=\emptyset$, but $\Pi_{0} \neq \emptyset$, then $\xi=-\alpha$ by Lemma 4.2. By Lemma 4.1(ii), the Laplace transform $Z(s)$ is unbounded on $(\xi, \infty)$, and $B_{1}>0$. The expression for $Z(s)$ in this case is given by (4.3). Recall that function $V(s+\alpha)$ is bounded on $(-\alpha, \infty)$ by Lemma 3.1(i). Therefore

$$
b=\lim _{s \rightarrow-\alpha+}(s+\alpha)^{2}\left[\left(\frac{A_{1}}{s+\alpha}+\frac{B_{1}}{(s+\alpha)^{2}}\right) w_{1}(p)+V(s+\alpha)\right]=B_{1} w_{1}(p)>0 .
$$

If $\Pi_{-} \neq \emptyset$, then $\xi=\alpha_{1}-\alpha$ by Lemma 4.2. By Lemma 4.1(i), the Laplace transform $Z(s)$ is unbounded on $(\xi, \infty)$, and $A_{1}>0$. The expression for $Z(s)$ is given by (3.4). Therefore

$$
\begin{aligned}
b=\lim _{s \rightarrow \alpha_{1}-\alpha+}\left(s+\alpha-\alpha_{1}\right)^{2}\left[\sum _ { k \in \Pi _ { - } } \left(\frac{A_{k}}{s+\alpha-\alpha_{k}}\right.\right. & \left.+\frac{B_{k}}{s+\alpha+\alpha_{k}}\right) w_{k}(p) \\
& \left.+\left(\frac{A_{m}}{s+\alpha}+\frac{B_{m}}{(s+\alpha)^{2}}\right) w_{m}(p)+V(s+\alpha)\right]=0 .
\end{aligned}
$$

Thus, the boundedness of $Z(s)$ on $(\xi, \infty)$, and the value of $b$ give us the following three distinct possibilities.

(i) $Z(s)$ is bounded on $(\xi, \infty)$. This can happen only if $\Pi_{-}=\Pi_{0}=\emptyset$. Therefore, in this case $\alpha=-\xi$. By Lemma 4.1(iii), $A_{1}>0$, and $z_{p}(t)=e^{-\alpha t} v(t, p)$. To determine the other two parameters $\beta$, and $\gamma$, consider the Fourier transform of $v(t, p)=e^{\alpha t} z_{p}(t)$. It is given by formula (3.5).

We have $A_{1}>0$, and $A_{2}=\left(g, w_{2}\right) \neq 0$ by the assumption. Therefore the first two positive singularities of the distribution $\mathcal{F} v$ are at $w=\alpha_{1}=\sqrt{\beta+\gamma-\alpha^{2}}$ and at $w=\alpha_{2}=\sqrt{4 \beta+\gamma-\alpha^{2}}$. Thus the unique identification for the parameters is given by

$$
\alpha=-\xi, \quad \beta=\frac{1}{3}\left(\alpha_{2}^{2}-\alpha_{1}^{2}\right), \quad \gamma=\alpha_{1}^{2}-\beta+\alpha^{2} .
$$

The conclusion is that if $Z(s)$ is bounded on $(\xi, \infty)$, then the parameter $q \in P$ in $z_{p}(t)=y(t, p ; q)$ is identifiable.

(ii) $Z(s)$ is unbounded on $(\xi, \infty)$, and $b \neq 0$. As shown above, this can happen only if $\Pi_{-}=\emptyset$, but $\Pi_{0} \neq \emptyset$. By Lemma 4.2, $\xi=-\alpha$. By Lemma 4.1(ii), $\Pi_{0}=\{1\}, A_{1}>0, B_{1}>0$. Therefore $\beta+\gamma=\alpha^{2}$. Since $\alpha=-\xi$ is already found, it gives the first equation for the sum $\beta+\gamma$.

By (4.6), $b=B_{1} w_{1}(p)$. Then the expression for the Laplace transform (4.3) can be used to find

$$
a=\lim _{s \rightarrow-\alpha+}(s+\alpha)\left(Z(s)-\frac{b}{(s+\alpha)^{2}}\right)=A_{1} w_{1}(p) \neq 0 .
$$


Therefore, by Lemma 4.1(ii)

$$
v(t, p)=e^{\alpha t} z_{p}(t)-(a+b t) .
$$

The first positive singularity of the Fourier transform $\mathcal{F} v$ occurs at $\alpha_{2}=\sqrt{4 \beta+\gamma-\alpha^{2}}$. Thus $4 \beta+\gamma=\alpha_{2}^{2}$. Together with the previously found equation $\beta+\gamma=\alpha^{2}$ this gives the unique identification

$$
\alpha=-\xi, \quad \beta=\frac{1}{3}\left(\alpha_{2}^{2}-\alpha^{2}\right), \quad \gamma=\alpha^{2}-\beta .
$$

(iii) The last case to consider is when $Z(s)$ is unbounded on $(\xi, \infty)$, and $b$, defined in (4.5), satisfies $b=0$. This implies $\Pi_{-} \neq \emptyset$. By Lemma 4.1(i), $1 \in \Pi_{-}, 0<\alpha_{1}<\alpha, A_{1}>0, B_{1}<0$. By Lemma $4.2, \xi=\alpha_{1}-\alpha$. Now we can find $\alpha$, and $\alpha_{1}$ as follows.

Define

$$
a_{1}=\lim _{s \rightarrow\left(\alpha_{1}-\alpha\right)+} Z(s)\left(s-\alpha_{1}+\alpha\right) .
$$

From (3.4) we conclude that $a_{1}=A_{1} w_{1}(p)$. Since $w_{1}(p)>0$, and $a_{1}$ is found, it gives us the value of $A_{1}$. On the other hand, by Theorem 2.2

$$
A_{1}=\frac{1}{2}\left(1+\frac{\alpha}{\alpha_{1}}\right)\left(g, w_{1}\right)+\frac{1}{2 \alpha_{1}}\left(h, w_{1}\right) .
$$

Since $\left(g, w_{1}\right)>0,\left(h, w_{1}\right) \geq 0$, and $0<\alpha_{1}<\alpha$, we conclude that $A_{1}>\left(g, w_{1}\right)$. Substitute $\alpha=\alpha_{1}-\xi$ into the equation for $A_{1}$, and find

$$
\alpha_{1}=\frac{1}{2} \frac{\left(h, w_{1}\right)-\xi\left(g, w_{1}\right)}{A_{1}-\left(g, w_{1}\right)} .
$$

Thus $\alpha_{1}$ and $\alpha=\alpha_{1}-\xi$ are found.

Now we eliminate the leading exponent from the expression (3.1) for $z_{p}(t)$. For $t \geq 0$ let

$$
r(t)=e^{\left(\alpha-\alpha_{1}\right) t} z_{p}(t)-a_{1} .
$$

By (3.1)

$$
\begin{aligned}
r(t)=B_{1} e^{-2 \alpha_{1} t} w_{1}(p)+e^{-\alpha_{1} t} \sum_{k \in \Pi_{-} \backslash\{1\}}\left(A_{k} e^{\alpha_{k} t}+\right. & \left.B_{k} e^{-\alpha_{k} t}\right) w_{k}(p) \\
& +e^{-\alpha_{1} t}\left(A_{m}+t B_{m}\right) w_{m}(p)+e^{-\alpha_{1} t} v(t, p) .
\end{aligned}
$$

The analysis of $r(t)$ is done in the same manner as the one for $Z(s)$ considered above. Specifically, first we prove the existence of $\xi_{1} \in \mathbb{R}$ such that $\left\|e^{-\eta t} r(t)\right\|_{\infty}<\infty$ for any $\eta>\xi_{1}$, and $\left\|e^{-\eta t} r(t)\right\|_{\infty}=$ $\infty$ for any $\eta<\xi_{1}$. Then the Laplace transform $R(s)$ of $r(t)$ is defined for $s>\xi_{1}$. Our goal is to determine whether $2 \in \Pi_{-}, 2 \in \Pi_{0}$, or $2 \in \Pi_{+}$, depending on the behavior of $R(s)$. If $2 \in \Pi_{+}$, then $\Pi_{0}=\emptyset$, and

$$
r(t)=B_{1} e^{-2 \alpha_{1} t} w_{1}(p)+e^{-\alpha_{1} t} v(t, p) .
$$

Therefore $\xi_{1}=-\alpha_{1}$, and $R(s)$ is bounded on $\left(\xi_{1}, \infty\right)$.

If $2 \in \Pi_{0}$, then

$$
r(t)=B_{1} e^{-2 \alpha_{1} t} w_{1}(p)+e^{-\alpha_{1} t}\left(A_{2}+t B_{2}\right) w_{2}(p)+e^{-\alpha_{1} t} v(t, p) .
$$

By Theorem 2.2, $A_{2}=\left(g, w_{2}\right)$. By the assumptions (4.1) or (4.2), $A_{2} \neq 0$. Therefore, irrespective of $B_{2}$, the term $e^{-\alpha_{1} t}\left(A_{2}+t B_{2}\right) w_{2}(p)$ is nontrivial. Thus we have $\xi_{1}=-\alpha_{1}$, and function $R(s)$ is unbounded on $\left(\xi_{1}, \infty\right)$. 
If $2 \in \Pi_{-}$, then $\xi_{1}=-\alpha_{1}+\alpha_{2}$, and $R(s)$ is unbounded on $\left(\xi_{1}, \infty\right)$. Note that $0<\alpha_{2}<\alpha_{1}$, since $\alpha_{k}=\sqrt{\alpha^{2}-\beta k^{2}-\gamma}$ for $k \in \Pi_{-}$. In this case $\xi_{1}>-\alpha_{1}$.

This investigation leads to the following three sub-cases of (iii).

(iii-1). $R(s)$ is bounded on $\left(\xi_{1}, \infty\right)$. Then the implication is that $\Pi_{-}=\{1\}, \Pi_{0}=\emptyset, 2 \in \Pi_{+}$, and $\xi_{1}=-\alpha_{1}$. Equation (4.10) gives $v(t, p)=e^{\alpha_{1} t} r(t)-B_{1} e^{-\alpha_{1} t} w_{1}(p)$. The first positive singularity of the Fourier transform of $v(t, p)$ is at $\alpha_{2}=\sqrt{4 \beta+\gamma-\alpha^{2}}$. At this point we know $\alpha_{1}-\alpha=\xi$, and $\alpha_{1}=-\xi_{1}$. We have $\alpha_{1}^{2}=\alpha^{2}-\beta-\gamma$, and $\alpha_{2}^{2}=4 \beta+\gamma-\alpha^{2}$. Therefore

$$
\alpha_{1}=-\xi_{1}, \quad \alpha=-\xi_{1}-\xi, \quad \beta=\frac{1}{3}\left(\alpha_{1}^{2}+\alpha_{2}^{2}\right), \quad \gamma=\alpha^{2}-\alpha_{1}^{2}-\beta .
$$

(iii-2). $R(s)$ is unbounded on $\left(\xi_{1}, \infty\right)$, and $\xi_{1}=-\alpha_{1}$. Then $\Pi_{-}=\{1\}$, and $\Pi_{0}=\{2\}$. Thus $4 \beta+\gamma-\alpha^{2}=0$. In this case we have $\alpha_{1}-\alpha=\xi, \alpha_{1}=-\xi_{1}, \alpha^{2}-\beta-\gamma=\alpha_{1}^{2}$, and $4 \beta+\gamma-\alpha^{2}=0$. Thus the parameters are uniquely identified by

$$
\alpha_{1}=-\xi_{1}, \quad \alpha=-\xi_{1}-\xi, \quad \beta=\frac{1}{3} \alpha_{1}^{2}, \quad \gamma=\alpha^{2}-\alpha_{1}^{2}-\beta .
$$

(iii-3). $R(s)$ is unbounded on $\left(\xi_{1}, \infty\right)$, and $\xi_{1} \neq-\alpha_{1}$. Then $1 \in \Pi_{-}$, and $2 \in \Pi_{-}$. Parameters $\alpha$ and $\alpha_{1}$ have already been determined above. In this case $\alpha_{2}=\xi_{1}+\alpha_{1}$. Since $\alpha_{1}^{2}=\alpha^{2}-\beta-\gamma$, and $\alpha_{2}^{2}=\alpha^{2}-4 \beta-\gamma$, this system can be solved to obtain

$$
\beta=\frac{1}{3}\left(\alpha_{1}^{2}-\alpha_{2}^{2}\right), \quad \gamma=\alpha^{2}-\alpha_{1}^{2}-\beta .
$$

Thus the parameters $(\alpha, \beta, \gamma)$ are uniquely identifiable from $z_{p}(t)$ in all cases.

\section{Computational algorithms}

A numerical implementation of the theoretical method described in Theorem 4.3 does not produce satisfactory identification results. Therefore, we choose to approach the parameter identification as the best fit to data problem

$$
J\left(q^{*}\right)=\inf _{P} J(q)=\inf _{P}\left\|y(q)-z_{d}\right\|_{L^{2}(Q)}^{2}
$$

described in the Introduction.

\section{Direct minimization (DM)}

Let $N>0$. Given $q=(\alpha, \beta, \gamma) \in P$ define the objective function

$$
J_{N}(q)=\left\|z_{d}(t)-y_{N}(t, p ; q)\right\|_{L^{2}(0, T)}^{2} .
$$

For the linearized sine-Gordon problem (1.2) the approximate solution $y_{N}(t, p ; q)$ is computed as in Theorem 2.2. For the nonlinear sine-Gordon problem (1.1) the approximate solution $y_{N}(t, p ; q)$ is computed numerically, see [6].

The identifiability analysis of the previous sections did not depend on the boundedness of the admissible set $P$. However, for a minimization problem it is natural to assume that the admissible set $P$ is bounded. To avoid an introduction of an additional notation we just redefine $P$ as a bounded box in $\mathbb{R}^{3}$

$$
P=\left\{(\alpha, \beta, \gamma) \in \mathbb{R}^{3}: 0 \leq \alpha \leq \alpha_{\max }, \beta_{\min } \leq \beta \leq \beta_{\max }, 0 \leq \gamma \leq \gamma_{\max }\right\} .
$$

The sought minimizer $q^{*}$ is found from

$$
q_{N}^{*}=\left(\alpha_{N}^{*}, \beta_{N}^{*}, \gamma_{N}^{*}\right)=\operatorname{argmin} J_{N}(\alpha, \beta, \gamma), \quad(\alpha, \beta, \gamma) \in P .
$$


The continuity of the solution map $y_{N}: P \rightarrow L^{2}(0, T ; H)$ for the sine-Gordon problem is established in [7]. The continuity proof remains valid for the linearized sine-Gordon problem. Therefore the minimization problem (5.4) has at least one solution. We can show that if $q^{*}$ is a limit point of the sequence $q_{N}^{*}, N=1,2, \ldots$ then $J\left(q^{*}\right)=\min _{q \in P} J(q),[6]$.

The nonlinear minimization is carried out by a modification of Powell's minimization method, [16]. The advantage of the Powell's method is that it does not require the knowledge of the derivatives (gradients) to minimize functions of several variables, but it still achieves a quadratic convergence near the points of minima.

The method iteratively minimizes a function of several variables. Here $q=(\alpha, \beta, \gamma)$, so the minimization is carried out in a three-dimensional space. Steps 1-7 describe one iteration of the method.

\section{Powell's minimization method}

1. Initialize the set of directions $u_{i} \in \mathbb{R}^{3}$ to the standard basis vectors $\mathrm{e}_{i}$ in $\mathbb{R}^{3}$

$$
u_{i}=\mathrm{e}_{i}, \quad i=1,2,3 .
$$

2. Save your starting position as $q_{0} \in \mathbb{R}^{3}$.

3. For $i=1,2,3$ move from $q_{0}$ along the direction $u_{i}$ and find the point of minimum $p_{i}$.

4. Re-index the directions $u_{i}$, so that (for the new indices) $J_{N}\left(p_{1}\right) \leq J_{N}\left(p_{2}\right) \leq J_{N}\left(p_{3}\right) \leq J_{N}\left(q_{0}\right)$.

5. Move from $q_{0}$ along the new direction $u_{1}$ and find the point of minimum $r_{1}$. Move from $r_{1}$ along the direction $u_{2}$ and find the point of minimum $r_{2}$. Move from $r_{2}$ along the direction $u_{3}$ and find the point of minimum $r_{3}$.

6. Set $v=r_{3}-q_{0}$.

7. Move from $q_{0}$ along the direction $v$ and find the minimum. Call it $q_{0}$. It replaces $q_{0}$ from step 2 .

8. Repeat the above steps until a stopping criterion is satisfied.

The minimization is further restricted to the admissible set $P \subset \mathbb{R}^{3}$, satisfying (5.3).

\section{Numerical results}

Let $T>0$. Choose $M \in \mathbb{N}$. Define the time grid $t_{j}=j M / T, j=0,1,2, \ldots, M$. The admissible set of parameters $q=(\alpha, \beta, \gamma)$ is taken to be

$$
P=[0,1] \times[0.2,1] \times[0,1] .
$$

We consider three numerical examples for the identifiability and the identification problems. Example 6.1 is for a linearized sine-Gordon equation. Two other examples are for a non-linear sine-Gordon equation.

The values $T=8, M=128, N=20$, and the test parameters

$$
q_{\text {test }}=(0.512,0.934,0.230) \in P
$$

are common in all the examples. The observation points are defined by $p_{j}=j \pi / 16: j=1,2, \cdots, 15$. Given $p \in(0, \pi)$ the simulated data $z_{d}(t)=y_{40}\left(t, p ; q_{\text {test }}\right)$ is generated by solving the appropriate linearized, or non-linearized sine-Gordon problem.

In every example the identification was obtained by the Direct minimization utilizing Powell's minimization method as described in section 5. The initial approximation $q_{0} \in P$ was chosen to be

$$
q_{0}=(0.5000,0.6000,0.5000)
$$

Example 6.1. The governing system is the linearized sine-Gordon problem $(2.2)$ with $g(x)=x^{2}(\pi-x)$ and $h(x)=x(\pi-x)$. 
Table 1 shows the identified parameters $q_{N}^{*}$ and the corresponding values of the objective function $J_{N}$ using different observations $z_{p_{j}}(t)$ with various observation points $p_{j}$. The table shows that almost precise identification was achieved by utilizing 12 iterations of the Powell's minimization method. The run time for the code execution was less than 1 second for each identification.

TABLE 1. Identification results for linearized sine-Gordon equation

\begin{tabular}{cccc}
\hline$p$ & Iterations & $q_{N}^{*}$ & $J_{N}\left(q_{N}^{*}\right)$ \\
\hline$p_{1}$ & 12 & $(0.5120,0.9340,0.2300)$ & 0.000021882 \\
$p_{3}$ & 12 & $(0.5120,0.9340,0.2300)$ & 0.000016025 \\
$p_{5}$ & 12 & $(0.5120,0.9340,0.2300)$ & 0.000011622 \\
$p_{7}$ & 12 & $(0.5120,0.9340,0.2300)$ & 0.000008510 \\
$p_{9}$ & 12 & $(0.5120,0.9340,0.2300)$ & 0.000007003 \\
$p_{10}$ & 12 & $(0.5120,0.9340,0.2300)$ & 0.000010157 \\
$p_{12}$ & 12 & $(0.5120,0.9339,0.2301)$ & 0.000009177 \\
$p_{14}$ & 12 & $(0.5120,0.9339,0.2301)$ & 0.000007986 \\
\hline
\end{tabular}

Example 6.2. Homogeneous damped sine-Gordon system

$$
\begin{aligned}
& y_{t t}+2 \alpha y_{t}-\beta y_{x x}+\gamma \sin y=0, \quad(t, x) \in Q=(0,8) \times(0, \pi), \\
& y(t, 0)=y(t, \pi)=0, \quad t \in(0,8), \\
& y(0, x)=x^{2}(\pi-x), y_{t}(0, x)=x(\pi-x), \quad x \in(0, \pi) .
\end{aligned}
$$

This type of problems was considered by us in [6]. The test parameters $q_{\text {test }}$ and the corresponding target behavior $z_{d}(t)$ are the same as in example 6.1. The nonlinear minimization is carried out by the Powell's minimization method. The stopping criterion for this iterative process was chosen to be

$$
\frac{\left|J_{N}\left(q_{m}\right)-J_{N}\left(q_{m-1}\right)\right|}{\left|J_{N}\left(q_{0}\right)\right|}<10^{-6} .
$$

Let $q_{N}^{*}=q_{m}$ be the set of parameters attained when the Powell's minimization method was terminated according to the stopping criterion (6.2). The identification results are shown in Table 2. It shows that the identification is successful for observations taken at various observation points $p_{j}$. The execution time for the sine-Gordon problem is about 22 seconds.

TABLE 2. Identification results for damped sine-Gordon equation (6.1)

\begin{tabular}{cccc}
\hline$p$ & Iterations & $q_{N}^{*}$ & $J_{N}\left(q_{N}^{*}\right)$ \\
\hline$p_{1}$ & 6 & $(0.51200,0.93340,0.23026)$ & 0.000043 \\
$p_{3}$ & 10 & $(0.51201,0.93402,0.23108)$ & 0.000033 \\
$p_{5}$ & 4 & $(0.51200,0.93400,0.22998)$ & 0.000000 \\
$p_{7}$ & 5 & $(0.51200,0.93400,0.23081)$ & 0.000000 \\
$p_{9}$ & 7 & $(0.51204,0.93400,0.23078)$ & 0.000000 \\
$p_{10}$ & 7 & $(0.51202,0.93400,0.23042)$ & 0.000000 \\
$p_{12}$ & 6 & $(0.51199,0.93400,0.23063)$ & 0.000000 \\
$p_{14}$ & 6 & $(0.51199,0.93400,0.23074)$ & 0.000000 \\
\hline
\end{tabular}

Example 6.3. Non-homogeneous sine-Gordon equation (6.1) with a forcing term $f=1$. Other assumptions are the same as in Example 6.2. 
This example is similar to examples considered in [6]. The identification results are shown in Table 3. It shows that the identification is successful for such more general equations. The execution time was about 22 seconds. A discussion of the performance of the method applied to noisy data can be found in $[6]$.

TABLE 3. Identification results for damped sine-Gordon equation (6.1) with a forcing term

\begin{tabular}{cccc}
\hline$p$ & Iterations & $q_{N}^{*}$ & $J_{N}\left(q_{N}^{*}\right)$ \\
\hline$p_{1}$ & 8 & $(0.51200,0.93399,0.23017)$ & 0.000035 \\
$p_{3}$ & 6 & $(0.51208,0.93403,0.23182)$ & 0.000117 \\
$p_{5}$ & 5 & $(0.51200,0.93400,0.23010)$ & 0.000000 \\
$p_{7}$ & 5 & $(0.51198,0.93400,0.23054)$ & 0.000000 \\
$p_{9}$ & 4 & $(0.51200,0.93400,0.22992)$ & 0.000000 \\
$p_{10}$ & 4 & $(0.51200,0.93400,0.23001)$ & 0.000000 \\
$p_{12}$ & 4 & $(0.51201,0.93400,0.23005)$ & 0.000000 \\
$p_{14}$ & 4 & $(0.51199,0.93390,0.23018)$ & 0.000000 \\
\hline
\end{tabular}

\section{Conclusions}

The identifiability problem for nonlinear damped sine-Gordon equation is an open problem. In this paper we have established the identifiability for linearized damped sine-Gordon problem. The results are also applicable to linearized Klein-Gordon equation since it has the same form.

Our method consists of a careful analysis of the Laplace and Fourier transforms of the observation $z_{p}(t), t>0$ of the system, conducted at a single point $p \in(0, \pi)$. Clearly, the results are also valid on any other interval $I \subset \mathbb{R}$. In this work the analysis is carried out for equations with constant coefficients, zero boundary conditions, and with no forcing term. The question whether the system parameters can be identified from the observation $z_{p}(t)$, when the observation interval for $t$ is bounded, remains open.

Numerical results based on the best fit to data method confirm that the identification is unique for a wide choice of initial approximation $q_{0}$ for the sought parameters $q_{t e s t}$.

We have also compared the obtained numerical results with experiments conducted in [6], where the identification was carried out for nonlinear sine-Gordon system. The numerical results show that the identification for such system is qualitatively the same as for the linearized one. That is, the numerical computation identifies the unique set of parameters $q^{*}$ which is practically identical to the test set $q_{\text {test }}$. Therefore, from the numerical evidence we can conclude that the nonlinear sine-Gordon problem may be identifiable under the same conditions as for the corresponding linearized problem.

Acknowledgements. The authors would like to thank the reviewer for valuable suggestions.

\section{References}

[1] A. R. Bishop, K. Fesser, P. S. Lomdahl. Influence of solitons in the initial state on chaos in the driven damped sine-Gordon system. Physica D, 7 (1983), 259-279.

[2] N. Dunford, J. Schwartz. Linear operators, Part I. Wiley \& Sons, New York, NY. Reprint of the 1958 original, 1988.

[3] L. C. Evans. Partial differential equations. Graduate studies in Mathematics, vol. 19. American Mathematical Society, Providence, R.I, 1998.

[4] S. Gutman, J. Ha. Identifiability of piecewise constant conductivity in a heat conduction process. SIAM J. Control and Optimization, 46(2) (2007), 694-713.

[5] S. Gutman, J. Ha. Parameter identifiability for heat conduction with a boundary input. Math. Comp. Simul., 79 (2009), 2192-2210.

[6] J. Ha, S. Gutman. Optimal parameters for a damped sine-Gordon equation. J. Korean Math. Soc., 46 (2009), 11051117. 
[7] J. Ha, S. Nakagiri. Identification of constant parameters in perturbed sine-Gordon equations. J. Korean Math. Soc., 43(5) (2006), 931-950.

[8] V. Isakov. Inverse problems for partial differential equations. Second edition. Applied Mathematical Sciences, vol. 127. Springer, New York, 2006.

[9] S. Kitamura, S. Nakagiri. Identifiability of spatially-varying and constant parameters in distributed systems of parabolic type. SIAM J. Control and Optimization, 15 (1977), 785-802.

[10] M. Levi. Beating modes in the Josephson junction. Chaos in Nonlinear Dynamical Systems, J. Chandra (Ed.) (1984), SIAM, Philadelphia.

[11] J. L. Lions. Optimal control of systems governed by partial differential equations. Springer-Verlag, New York, 1971.

[12] A. C. Metaxas, R. J. Meredith. Industrial microwave heating. Peter Peregrinus, London, 1993.

[13] S. Nakagiri. Review of Japanese work of the last 10 years on identifiability in distributed parameter systems. Inverse Problems, 9(2) (1993), 143-191.

[14] R. Ortega, A. M. Robles-Perez. A maximum principle for periodic solutions of the telegraph equation. J. Math. Anal. Appl., 221 (1998), 625-651.

[15] A. Pierce. Unique identification of eigenvalues and coefficients in a parabolic problem. SIAM J. Control and Optimization, 17(4) (1979), 494-499.

[16] W. H. Press, S. A. Teukolsky, W. T. Vetterling, B. P. Flannery. Numerical Recepies in FORTRAN, 2nd ed. Cambridge University Press, Cambridge, 1992.

[17] N. F. Smyth, A. L. Worthy. Soliton evolution and radiation loss for the sine-Gordon equation. Physical Reviews E, (1999), 2330-2336.

[18] M. Taylor. Partial differential equations I. Basic theory. Second edition. Applied Mathematical Sciences, vol. 115. Springer, New York, 2011.

[19] R. Temam. Infinite-Dimensional Dynamical Systems in Mechanics and Physics, 2nd ed. Applied Mathematical Sciences, vol. 68, Springer-Verlag, 1997.

[20] K. Yosida. Functional Analysis, 6th ed. Springer-Verlag, 1980. 